TRABAJADORES DE LA CONSTRUCCIÓN EN COSTA RICA Y SU CONSUMO DE MEDIOS DE COMUNICACIÓN.

\title{
Trabajadores de la Construcción en Costa Rica y su Consumo de Medios de Comunicación
}

\section{Constructions Workers in Costa Rica and their Media Consumption}

Pierre Murray Kattia*, Barrantes Dall Anese Julia**

*Máster en Ciencias de la Comunicación y Periodismo. University of Kansas. Docente e Investigadora de la Escuela de Ciencia de la Comunicación Colectiva de la Universidad de Costa Rica. San José, Costa Rica. ORCID: https://orcid.org/0000-0002-4383-7324.

**Máster en Administración de Empresas. Universidad de Durham. Licenciada en Ciencias de la Comunicación Colectiva con Énfasis en Publicidad. Universidad de Costa Rica. Email: calim@ucr.ac.cr. ORCID: https://orcid.org/0000-0002-7974-8008.

Correo para recibir correspondencia: kattia@ucr.ac.cr 
TRABAJADORES DE LA CONSTRUCCIÓN EN COSTA RICA Y SU CONSUMO DE MEDIOS DE COMUNICACIÓN.

\section{RESUMEN}

OBJETIVO: Conocer y analizar los gustos, hábitos y preferencias de consumo de medios de comunicación de los Trabajadores de la Construcción en Obra (TCO) como referente para el diseño y ejecución de estrategias de comunicaciones integradas de marketing dirigidas a este segmento.

MATERIAL Y MÉTODO: Se realizaron dos grupos focales y se aplicó un cuestionario a una muestra de cuarenta y seis personas. Como caso de estudio se examinó el posicionamiento del periódico Manos a la Obra de la Cámara Costarricense de la Construcción. Para el análisis de resultados se utilizó el modelo denominado cadena de medios-fines.

RESULTADOS: Se encontró que la televisión y el periódico impreso son los medios preferidos, y que su consumo está asociado a la esfera doméstica y al espacio laboral respectivamente. La evaluación de Manos a la Obra fue positiva. La muestra identifica como atributos deseables un medio escrito, de extensión corta, que aborde temas de forma escalonada y que refleje los valores del gremio.

CONCLUSIONES: Los TCO evidencian conexiones entre los atributos tangibles que prefieren en un medio de comunicación, sus beneficios percibidos y los valores profundos que comparten con su grupo de referencia. El mayor desafío encontrado es la distribución y la sustentabilidad económica del medio de comunicación.

PALABRAS CLAVE: Ingeniería de la construcción. Consumo. Medios de comunicación.

\section{ABSTRACT}

OBJECTIVE: To know and analyze the likes, habits, and preferences of media consumption of Construction Workers, in Construction Site (TCO, in its Spanish acronym), as a benchmark for the design and execution of integrated marketing communications strategies aimed at this segment.

MATERIAL AND METHOD: Two focus groups were conducted, and a questionnaire was applied to a sample of forty-six people. As a case study, the positioning of Manos a la Obra newspaper of the Costa Rican Construction Chamber was examined. For the analysis of results, the meansend chain model was used. 
TRABAJADORES DE LA CONSTRUCCIÓN EN COSTA RICA Y SU CONSUMO DE MEDIOS DE COMUNICACIÓN.

RESULTS: It was found that television and printed newspaper are the preferred media, and that their consumption is associated with the domestic sphere and the workplace, respectively. The evaluation of Manos a la Obra was positive. The sample identifies as desirable attributes a written medium, of short length, that addresses issues in a staggered manner and that reflects the values of the union.

CONCLUSIONS: TCOs show connections among the tangible attributes they prefer in a communication medium, their perceived benefits, and the deep values they share with their peer group. The greatest challenge encountered is the distribution and economic sustainability of the communication medium.

KEY WORDS: Construction engineering. Consumption. Media.

\section{INTRODUCCIÓN}

Los Trabajadores de la Construcción en Obra (en adelante TCO) tienen un rol fundamental en su industria, pues son quienes materializan los proyectos. Sus decisiones influyen sobre el diseño de la obra, así como en la selección de las técnicas y la calidad de los materiales utilizados. Por ende, su labor tiene efecto directo sobre el consumo del ramo, sobre los presupuestos asignados, la seguridad y el bienestar de los usuarios de las edificaciones.

Según se desprende de las entrevistas realizadas en la etapa exploratoria, la mayoría de los TCO carecen de educación formal básica, y por lo general, aprenden su oficio bajo la supervisión de un mentor. Este sistema de aprendizaje favorece la transmisión del conocimiento sobre materiales y técnicas de una generación a otra, pero puede igualmente llegar a impedir o retardar la adopción de prácticas más seguras o novedosas.

Personas clave de la Asociación Costarricense de Maestros de Obra (ACMO) y la Cámara Costarricense de la Construcción (CCC), manifestaron la necesidad de contar con un estudio sobre medios de comunicación, que guiara la creación de espacios mediáticos apropiados para el segmento (J. Alvarado, comunicación personal, mayo 2010; A. Durmond, comunicación personal, enero 2011; D. González, comunicación personal, abril 2011 y R. Bonilla, comunicación personal, abril 2012). En efecto, al momento del estudio circulaban en Costa Rica dos 
TRABAJADORES DE LA CONSTRUCCIÓN EN COSTA RICA Y SU CONSUMO DE MEDIOS DE COMUNICACIÓN.

publicaciones dirigidas a este segmento, financiados y apoyados por los miembros del gremio, que enfrentaban dificultad para sobrevivir (R. Bonilla, comunicación personal, abril 2012).

En la revisión bibliográfica, no se encontraron estudios académicos o comerciales que analizaran las necesidades y preferencias mediáticas de esta población. Surgió así la pregunta: ¿Cuáles son los gustos, hábitos y preferencias de consumo de medios de comunicación de los TCO que podrían ser usados como referente para el diseño y la ejecución de estrategias de comunicaciones integradas de marketing dirigidas a este segmento?

\section{MARCO TEÓRICO}

Las investigaciones académicas sobre los TCO se enfocan mayormente en su salud ocupacional y en la prevención de riesgos laborales desde la perspectiva del economicismo. No obstante, algunos autores incorporan a su análisis ciertos factores propios de las ciencias sociales como variable causal para los riesgos de salud. Entre estos, Baruah (2010), Zhou y Zhou (2009), lacuone (2005) y Baldi y Obando (1998) encontraron que el género y la condición migratoria influyen sobre la situación laboral y el incremento en la exposición a riesgos en la obra. Por su parte, Cunradi, Ames y Moore (2008) hallaron causalidad entre los factores laborales y el índice de violencia intrafamiliar en una muestra de TCO.

Diversos estudios han analizado lo que identifican como un incipiente uso de redes sociales en la industria de la construcción (Tang, Griffith, Stevens, y Hardie, 2020; Azhar, S., Riaz, Z. y Robinson, D., 2019; Perera, Victoria y Brand, 2017; Azhar, S., y Abeln, J. M., 2014). Sin embargo, no se encontraron documentos que hicieran distinción entre perfiles ocupacionales.

La ACMO produce la revista Tecnocon, con el fin de promover la actualización de sus agremiados. Esta revista incluye novedades sobre técnicas y materiales, y repasa el contenido de las capacitaciones impartidas en sus talleres y charlas (Godínez, comunicación personal, marzo 2016). Por su parte, la CCC publicaba el periódico Manos a la Obra, cuyo contenido editorial era independiente (no pagado por patrocinadores) e incluía espacios comerciales pagados (J. Alvarado, Comunicación personal, mayo 2010). Al momento de la investigación, circulaban otras tres iniciativas mediáticas privadas dirigidas a este segmento cuyo contenido era enteramente patrocinado (Bonilla, comunicación personal, abril 2012). 
TRABAJADORES DE LA CONSTRUCCIÓN EN COSTA RICA Y SU CONSUMO DE MEDIOS DE COMUNICACIÓN.

\section{Fundamentación teórica del estudio}

En el marketing para la elaboración de las estrategias dirigidas a los grupos de consumidores, usualmente, se analizan dos tipos de factores que influyen en la toma de decisiones de las personas, los internos (pensamientos, creencias, sentimientos, etc.) y los estímulos del ambiente social y cultural (Kotler y Armstrong, 2013; Schiffman y Kanuk, 2005; Hawkins, Best y Coney, 2004; Alonso, 2001; y Loudon y Bitta, 1995).

Para este estudio, se utilizó el modelo de Peter y Olson (2006), que analiza los elementos internos y externos al individuo, en donde cada elemento puede actuar como detonante, así como efecto del otro y afectar la estrategia de marketing (Figura 1).

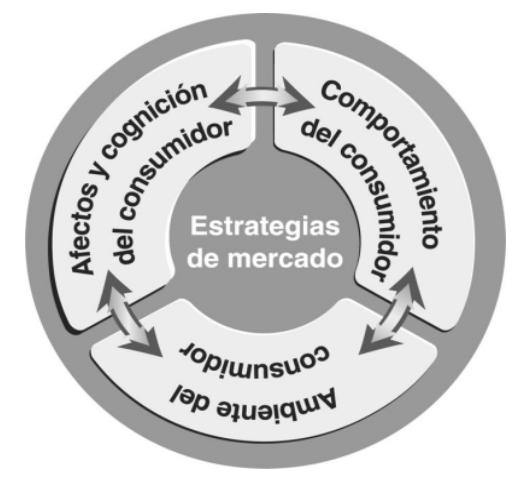

Figura 1. Elementos para el análisis del consumidor (Peter y Olson, 2006, p. 22).

Asimismo, con base en esta interacción de los factores los autores proponen el uso de la cadena de medios-fines en la cual se conjugan lo afectivo (emociones, estado de ánimo o actitudes) y lo cognitivo (pensamientos, significados, creencias, evaluaciones y el conocimiento).

En la cadena de medios-fines el consumidor evalúa los atributos que conoce del producto como un medio para alcanzar un fin, una consecuencia o un valor. El involucramiento del usuario con el producto dependerá de la relevancia que tenga este para su vida y la fuerza de las conexiones entre los medios y sus fines.

Peter y Olson (2006) identifican tres elementos que pueden influir sobre el comportamiento del consumidor hacia el producto:

a) Los atributos: las características tangibles o abstractas;

b) Los beneficios o riesgos: las consecuencias percibidas por su uso; 
TRABAJADORES DE LA CONSTRUCCIÓN EN COSTA RICA Y SU CONSUMO DE MEDIOS DE COMUNICACIÓN.

c) Los valores: los objetivos amplios del individuo (por ejemplo, seguridad o éxito).

Otro factor es el ambiente, que comprende estímulos físicos y sociales; y para ello proponen el estudio de la cultura y subcultura del individuo como parte de su ambiente. Por esto, es común que miembros de un mismo gremio o clase social consuman medios afines, que los identifican, a la vez que les permiten interactuar con sus pares.

Las decisiones de consumo se ven afectadas por los grupos de referencia, que tienen identidades simbólicas con valores, prioridades y comportamientos similares. En este contexto los productos funcionan como transmisores de significados culturales.

De manera que, un comportamiento de adquisición repetida sugiere una satisfacción con los atributos y beneficios percibidos en el servicio o producto (Peter y Olson, 2006).

También cabe señalar que los canales de difusión son intrínsecos al mensaje publicitario en la estrategia de marketing, pues es a través de ellos llega el mensaje a la audiencia o público objetivo (Pride y Ferrel, 1997).

\section{MATERIAL Y MÉTODO}

Con el fin de conocer y analizar los gustos, hábitos y preferencias de consumo de medios de comunicación de los TCO, se definieron tres objetivos específicos:

- Identificar los medios que utilizan para informarse y entretenerse.

- Reconocer los medios que utilizan para informarse sobre temas relacionados con su oficio y gremio.

- Determinar el posicionamiento del periódico Manos a la Obra de la CCC entre los TCO.

Se eligió como caso de análisis Manos a la Obra por ser el único medio diseñado y dirigido específicamente a este grupo e independiente en su línea editorial.

Se realizó una investigación exploratoria y descriptiva cualitativa. La investigación se enfocó en los aspectos ambiental-cognitivo y afectivo. La recolección de los datos se realizó a través de entrevistas y grupos focales (autoreporte) y no en la observación directa del comportamiento. 
TRABAJADORES DE LA CONSTRUCCIÓN EN COSTA RICA Y SU CONSUMO DE MEDIOS DE COMUNICACIÓN.

\section{Población}

En la revisión de la literatura, no se encontró una denominación común que designe a la población en estudio según la naturaleza de sus labores (Instituto Nacional de Estadística y Censos, 2011; Ministerio de Trabajo y Seguridad Social de Costa Rica, 2009; Unidad de Estadística de La Organización de las Naciones Unidas, 2009). De ahí, que las autoras proponen la siguiente definición: Trabajadores de la Construcción en Obra (TCO) son aquellos individuos que desempeñan funciones principalmente manuales, cuyo puesto de trabajo se encuentra en el campo de la construcción, que participan de la edificación y que no requieren educación formal como requisito para desempeñar sus funciones.

\section{Muestra}

Uno de los retos enfrentados fue la alta movilidad geográfica del segmento, debido a que suelen cambiar de residencia según la oferta de empleo y la ubicación geográfica de la obra. Además, ninguna organización pública o privada en Costa Rica posee datos detallados de las personas dedicadas a esta actividad.

El único listado de referencia disponible, al momento del estudio, era la lista de membresía de la ACMO, que contenía 130 nombres. Es evidente que este número no es representativo de la población real. Además, cada semana se integraban nuevos miembros y otros abandonaban la agrupación. Se optó por una muestra no probabilística y por criterio de expertos, debido a que "lo que se busca en la indagación cualitativa es profundidad. Por lo tanto, se pretende calidad en la muestra, más que cantidad" (Hernández, Fernández, y Baptista, 2006, p. 562). Solamente se incluyeron personas mayores de edad.

Se usó como principal contacto a la ACMO, al ser el mayor esfuerzo de organización y agremiación encontrado. Su principal actividad es la de fomentar la capacitación de sus integrantes por medio de alianzas con instituciones y de charlas semanales impartidas por empresas. Todo esto hace que puedan ser considerados como líderes dentro del gremio y, por lo tanto, influyentes para sus pares.

\section{Instrumentos de investigación}

Los datos se recolectaron utilizando un cuestionario y dos grupos focales. El cuestionario fue aplicado a 46 miembros de la ACMO, en dos sesiones. El primer grupo focal se realizó con ocho 
TRABAJADORES DE LA CONSTRUCCIÓN EN COSTA RICA Y SU CONSUMO DE MEDIOS DE COMUNICACIÓN.

miembros de la ACMO, identificados por la Junta Directiva como personas de alta participación y compromiso; el segundo se hizo con una cuadrilla de nueve trabajadores en los predios del proyecto constructivo Condominios del Río Invierno, luego de la jornada laboral.

\section{Categorías de análisis}

Para el análisis de los resultados se utilizaron las categorías:

a) hábitos de consumo de medios de comunicación, y;

b) gustos y preferencias.

La variable "hábitos" se define en la Real Academia Española como un "modo especial de proceder o conducirse adquirido por repetición de actos iguales o semejantes" (RAE, s.f., definición 1). En este caso, el estudio se centró en la descripción de un comportamiento reiterado de uso de medios de comunicación.

La variable "gustos y preferencias" combina la inclinación de una persona hacia algo particular con la elección activa que hace de ella. Los gustos se definen como la "propia voluntad, determinación o arbitrio" (RAE, s.f., definición 4) y las preferencias como la "elección de alguien o algo entre varias personas o cosas" (RAE, s.f., definición 2).

\section{RESULTADOS}

Los grupos focales revelaron que los medios de comunicación preferidos por los TCO para obtener información y entretenimiento son el periódico y la televisión. Un 89\% de las personas entrevistadas indicó ver Teletica Canal 7 y el 65\% ven Repretel Canal 6 (los principales canales de televisión abierta del país). Los demás canales, tanto de señal abierta como de cable, fueron mencionados por menos del $40 \%$ del total de la muestra.

En el caso del periódico, los resultados de hábitos de consumo muestran una preferencia hacia el Diario Extra, leído regularmente por un 74\% de quienes acostumbran comprar el periódico, mientras que La Teja es leído por el 65\% y La Nación por un 51\%, mientras que la radio y la revista no fueron consideradas relevantes para los participantes.

Como se puede observar, en las Figuras 2 y 3, los temas de mayor interés son las noticias y los deportes. En la programación televisiva con un 57\% y un 52\%, respectivamente, y en los periódicos con un $89 \%$ y un $65 \%$. 
TRABAJADORES DE LA CONSTRUCCIÓN EN COSTA RICA Y SU CONSUMO DE MEDIOS DE COMUNICACIÓN.

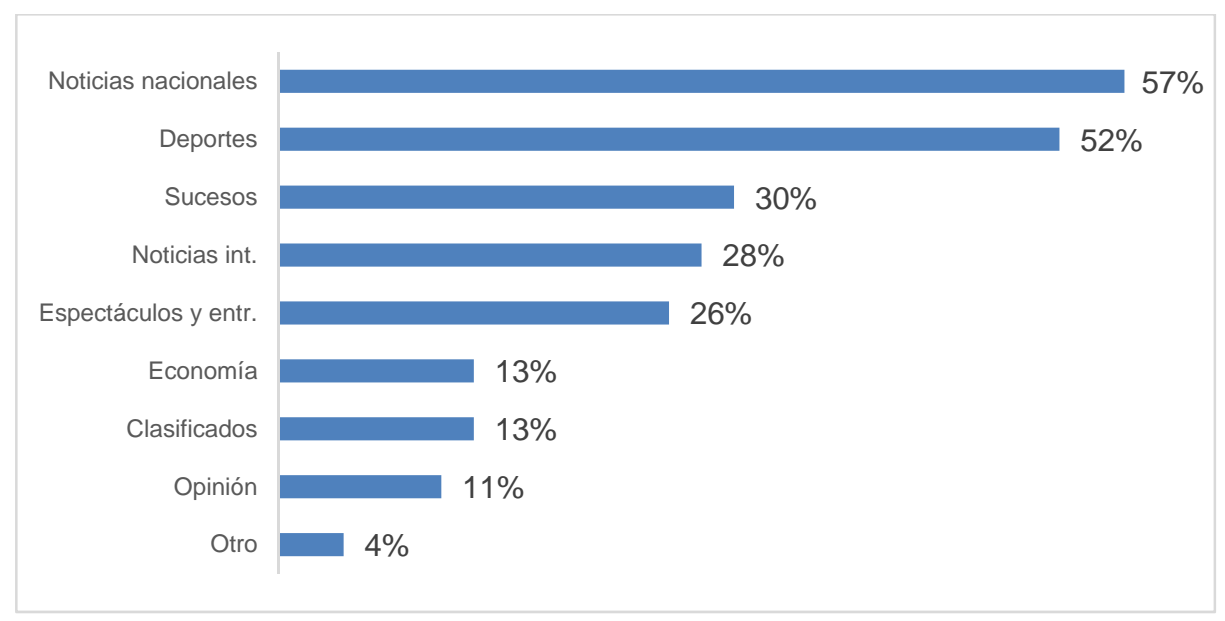

Figura 2. Tipos de programas de televisión que prefieren ver.

Fuente: Elaboración propia.

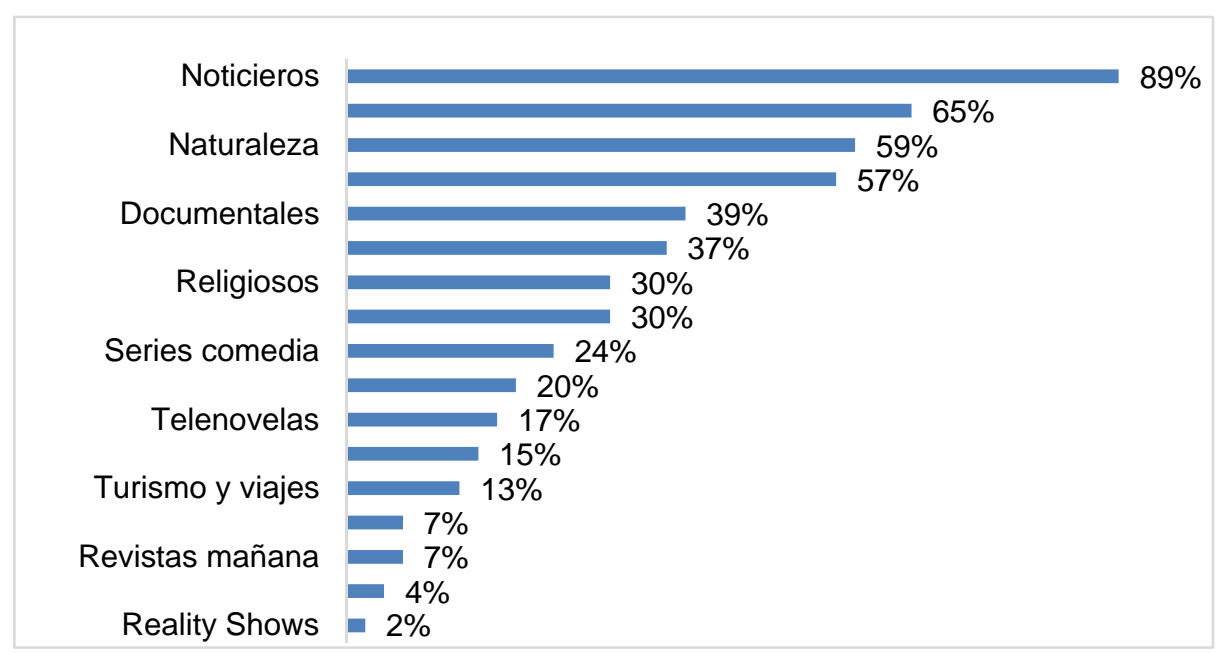

Figura 3. Secciones preferidas del periódico.

Fuente: Elaboración propia.

Los resultados reflejan que el uso de la televisión se da principalmente en el hogar. Como se muestra en la Figura 4, el 80\% de las personas entrevistadas indicaron ver televisión entre de las 6:00 p.m. a las 11:00 p.m. 
TRABAJADORES DE LA CONSTRUCCIÓN EN COSTA RICA Y SU CONSUMO DE MEDIOS DE COMUNICACIÓN.

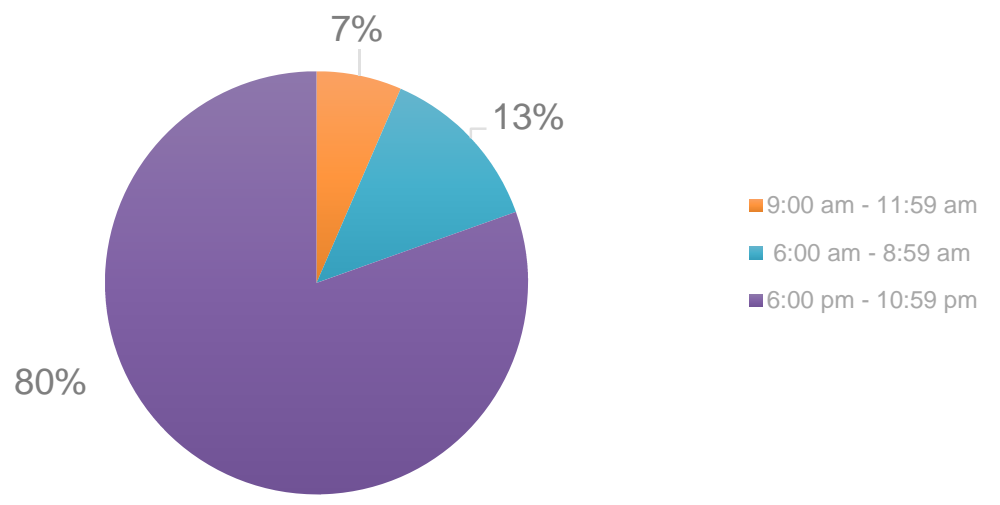

Figura 4. Horario en que acostumbra ver televisión.

Fuente: Elaboración propia.

Como se puede observar en la Figura 5, el 56.5\% suelen ver la televisión por un periodo promedio de una a dos horas al día y un $35 \%$ lo hace entre tres y cuatro horas.

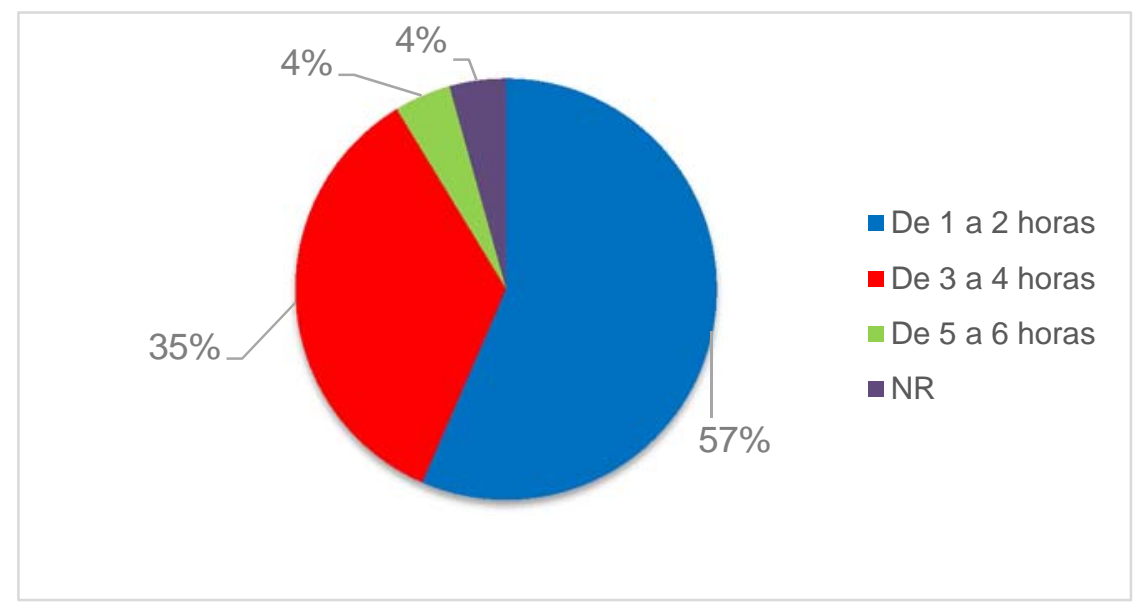

Figura 5. Cantidad de horas diarias dedicadas a la televisión.

Fuente: Elaboración propia.

Las respuestas de los grupos focales revelan que la atención a la televisión es limitada, pues los TCO suelen dividir este tiempo con sus familias. Además, los participantes hicieron constantes referencias al cansancio que experimentan después de una larga jornada de trabajo. Todo esto hace suponer que la televisión se asocia con el espacio doméstico, en donde reciben información y entretenimiento. 
TRABAJADORES DE LA CONSTRUCCIÓN EN COSTA RICA Y SU CONSUMO DE MEDIOS DE COMUNICACIÓN.

Por el contrario, se encontró una vinculación del periódico con el espacio laboral. Como se puede observar en la Figura 6, esto inicia con su compra, pues un 77\% de quienes afirmaron leerlo, lo adquieren en una pulpería (tienda de conveniencia) o en un quiosco. Los participantes de los grupos focales mencionaron que lo compran de camino a su trabajo y que lo leen en sus momentos de descanso. Algunos ejemplos de los comentarios al respecto se presentan en la Figura 7.

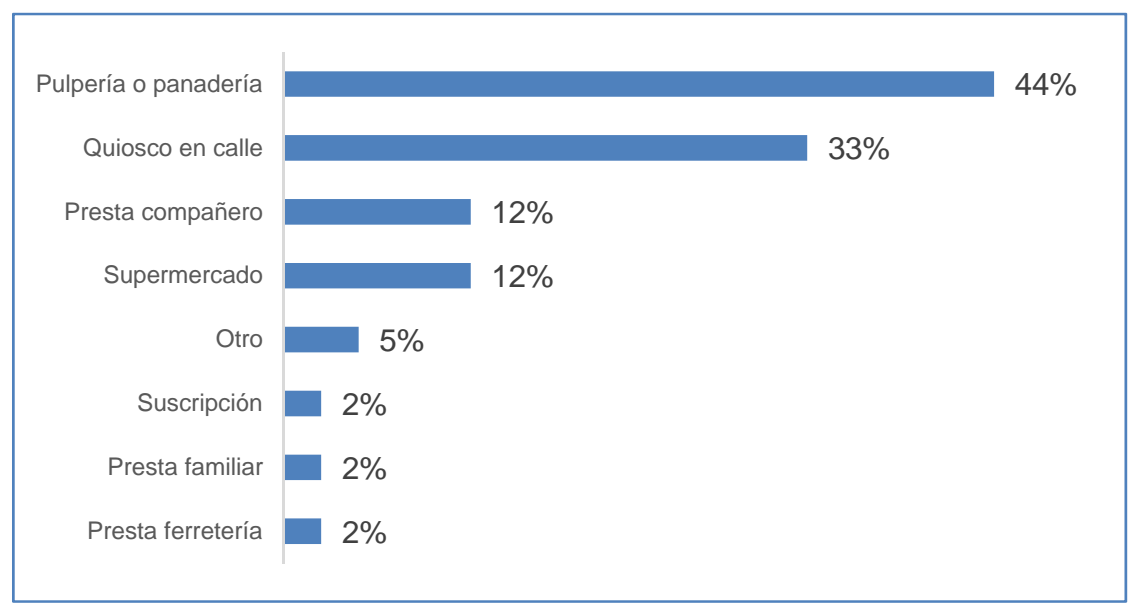

Figura 6. Sitio de adquisición del periódico.

Fuente: Elaboración propia.

Participante 1: Yo preferiría que fuera un periódico.

Participante 2: Sí, un periódico. En televisión normalmente si se le escapó ese detalle, esa información ya se fue.

Participante 3: Si no lo ve a la hora exacta que pasó, ya no... ya no.

Participante 4: En cambio esto (un periódico) usted lo tiene a cualquier hora, se lo puede pasar uno a un amigo también.

Participante 2: ¿Se interesó demasiado en la nota? Mejor la guarda y ahí la va a tener.

Figura 7. Ejemplo de respuestas obtenidas en grupo focal. Preferencias de medio de comunicación.

Fuente: Elaboración propia. 
TRABAJADORES DE LA CONSTRUCCIÓN EN COSTA RICA Y SU CONSUMO DE MEDIOS DE COMUNICACIÓN.

Se reconoció la identificación de clase social como una ventaja del periódico, específicamente del Diario Extra y La Teja. En contraste, el periódico La Nación es visto como ajeno, enfocado en temas financieros y vinculado a una clase ejecutiva que cuenta con un mayor poder adquisitivo.

\section{Atributos de un medio ideal dirigido específicamente a los TCO}

Los participantes de los grupos focales mostraron gran interés por un medio dedicado a ellos y aseguraron que sus colegas tendrían igual inclinación. Indicaron que su participación en las capacitaciones de la ACMO evidencia este interés, porque para asistir deben trasladarse hasta San José (distrito central de la capital), sacrificar el tiempo con sus familias y el descanso, así como invertir dinero en el transporte y la mensualidad. Además, mencionaron que la mayoría de los asociados han recibido cursos técnicos en la Universidad de Costa Rica y el Instituto Nacional de Aprendizaje.

Asimismo, los TCO acostumbran prácticas solidarias, como la de compartir información entre compañeros. Todo esto describe a un gremio interesado por mantenerse actualizado y con pocas fuentes disponibles para hacerlo.

Al preguntar por el mejor formato para recibir información, las respuestas fueron consistentes al ser la televisión y el medio escrito los elegidos por la mayoría. Sin embargo, hubo diferencias entre las respuestas en ambos instrumentos; el 48\% de quienes respondieron el cuestionario prefirieron la televisión, 33\% eligió la revista y apenas un 26\% prefirió el periódico. En contraste, los participantes de los grupos focales prefirieron el medio escrito y desaprobaron la televisión para tratar temas de construcción. Indicaron que, al ser un medio gráfico, su consumo no se vería afectado por los constantes ruidos presentes en los proyectos, como sucede con la radio o la televisión. Además, la portabilidad del periódico les permite compartir información de interés con sus compañeros y su permanencia les ofrece la posibilidad de revisarlo en detalle de forma espaciada, principalmente cuando se trata de artículos que merecen estudio.

Una persona manifestó la conveniencia de incluir el Internet que, según su criterio, ha ganado presencia, principalmente para la comunicación personal. Esta idea fue desechada por los otros participantes, que lo percibían como un medio de alto costo y asociado a una "clase ejecutiva".

Las características identificadas como atributos deseables en un medio de comunicación dirigido específicamente a TCO, son relativamente uniformes. Los resultados de los grupos focales coincidieron en que la extensión tanto de los artículos como del conglomerado final debe ser corta 
TRABAJADORES DE LA CONSTRUCCIÓN EN COSTA RICA Y SU CONSUMO DE MEDIOS DE COMUNICACIÓN.

e invitar a la lectura. Para obtener la profundidad necesaria en los temas, recomendaron hacer un abordaje escalonado de artículos cortos, que además cree expectativa sobre la siguiente edición.

Los integrantes de la muestra manifestaron que estar dispuestos a pagar por un medio escrito dirigido a ellos, un poco más de lo que desembolsan, usualmente, por los medios tradicionales que consumen. Normalmente invierten entre 300 y 400 colones (menos de 1 USD) por un ejemplar del periódico. También afirmaron estar dispuestos a pagar un sobreprecio en su periódico regular si este incluye un inserto con contenido relevante para su trabajo.

Los participantes de los grupos focales indicaron que adquieren información sobre sus temas de interés en otras fuentes como: revistas de bienes raíces y remodelación, el inserto de la Ferretería EPA y programas de televisión como Megaestructuras de National Geographic Channel, para mantenerse al día con su industria.

Otro tema que atrae a los TCO, es el de la oferta de empleo, debido a la alta rotación de personal que caracteriza a la industria de la construcción. También se sienten atraídos por información acerca de precios bajos y ofertas, tanto en herramientas y materiales de trabajo, como de bienes de consumo doméstico.

Finalmente, la mayor parte de las personas consultadas coincidieron en la pertinencia de incluir artículos de entretenimiento, principalmente referentes al fútbol. Esto coincide con los resultados del cuestionario de investigación, donde "deportes" fue el principal tema de interés luego de las noticias.

En las discusiones surgieron dos factores que merecen especial atención. Los Grupos de referencia afectan de manera transversal cualquier iniciativa de marketing dirigida a esta población y la Distribución se presenta como el principal desafío.

\section{Grupos de referencia}

Se advierte un autoconcepto de gremio claro y relativamente uniforme. También se percibe un gran respeto hacia las personas que ocupan cargos superiores, cuyos méritos se obtienen por su experiencia. 
TRABAJADORES DE LA CONSTRUCCIÓN EN COSTA RICA Y SU CONSUMO DE MEDIOS DE COMUNICACIÓN.

El esfuerzo es uno de los principales valores mencionados. Constantemente, se hizo referencia a su difícil horario laboral que los obliga a levantarse muy temprano en la mañana y regresar tarde a sus hogares.

Otro valor importante es el de la humildad, al reconocerse como una clase de bajos recursos económicos y luchadora, que enfrenta dificultad para adquirir bienes de consumo doméstico, materiales de trabajo e incluso conocimiento. Esto también estimula su compañerismo, que se expresa, entre otras formas, en el hábito de compartir información con sus colegas para incentivar el aprendizaje y superación mutua.

Los TCO también mostraron competencia y deseo de superación, debido a que su forma para ascender en la jerarquía laboral es mostrándose a sus superiores y destacando sobre sus compañeros en valores como su capacidad, aprendizaje y responsabilidad. Un participante del grupo focal lo ejemplifica al narrar su experiencia como peón:

"Diay, en el momento mío personal, cuando yo empecé a trabajar en la construcción, yo quería ser el primero. Aunque fuera volando pala, que aquel no me ganara haciendo un metro más de zanja. Y yo luchaba por eso". (Transcripción literal de grupo focal, 29 de julio del 2013)

\section{Distribución}

El trabajo en construcción se realiza usualmente por proyectos, lo que comúnmente implica un cambio frecuente de empleador y de residencia. Esto dificulta el acceso a los medios por suscripción. Por otra parte, los resultados muestran que existe un hábito de adquirir medios impresos en vía pública, en un quiosco (venta al pregón) o en una pulpería (tiendas de conveniencia). En efecto, al consultar a los TCO sobre este tema, destacó la buena distribución de los periódicos Diario Extra y La Teja y sugirieron utilizar los mismos mecanismos, ubicando el medio en puestos en la calle.

La figura del inserto también fue nombrada como una buena opción, al tomar como referencia el éxito percibido de la publicación de EPA en La Nación.

En el primer grupo focal se mencionaron las ferreterías como una buena opción de distribución; no obstante, posteriormente, recordaron su propia experiencia con la revista Tecnocon, que no fue aceptada en algunos locales por contener publicidad de ferreterías competidoras o de productos no disponibles en el establecimiento. Los participantes del segundo grupo focal 
TRABAJADORES DE LA CONSTRUCCIÓN EN COSTA RICA Y SU CONSUMO DE MEDIOS DE COMUNICACIÓN.

señalaron que los TCO que son empleados por empresas constructoras rara vez visitan las ferreterías, dado que los materiales son suplidos directamente por la empresa y que aquellos que trabajan por cuenta propia tampoco acostumbran visitarlas, pues los clientes prefieren hacer sus propias compras para confirmar la veracidad de los precios de los materiales adquiridos. Es así que, aunque no fue descartado, tampoco hubo una opinión fuerte a favor de las ferreterías como punto de distribución.

\section{Posicionamiento del periódico Manos a la Obra}

En ambos instrumentos se consultó a la muestra su opinión sobre el periódico Manos a la Obra. En los grupos focales, se entregó un ejemplar a cada participante para su valoración. La percepción del medio fue muy positiva. Las principales fortalezas identificadas hacen referencia a sus atributos funcionales. La elección del tipo de medio escrito. El uso de ilustraciones y fotografías para ejemplificar los procesos de construcción. El tono y el uso de un lenguaje sencillo, cotidiano, popular. La pertinencia de los artículos relacionados con nuevas técnicas, así como la presencia y el valor dado a la figura del TCO y a su conocimiento.

Los entrevistados recomendaron incorporar una sección en donde se enumeren los proyectos venideros o que se encuentran en proceso de contratación y se den consejos en materia de legislación laboral. También sugirieron una sección de ayuda al consumidor, que incluya publicidad tipo "shopper" con los precios de materiales, herramientas y artículos de la canasta básica.

En el estudio, la principal debilidad encontrada fue el desconocimiento generalizado de la existencia del periódico Manos a la Obra. La mayoría de las personas consultadas, no habían escuchado hablar él y quienes lo conocían habían leído pocos ejemplares. No conocido incluso entre las personas que laboraban en las empresas asociadas a la CCC, que fue la principal fuente de distribución de medio.

\section{DISCUSIÓN}

Se desprende de la investigación que los TCO tienen conexiones claras y evidentes entre a- los atributos tangibles que prefieren en un medio de comunicación (por ejemplo, portabilidad y diseño), b- los beneficios percibidos (por ejemplo, la capacidad de revisar la información sin distracciones) y c- los valores profundos que comparten con su grupo de referencia (por ejemplo, el deseo de superación). La Figura 8, ilustra el ejemplo a través del modelo de medios-fines. 
TRABAJADORES DE LA CONSTRUCCIÓN EN COSTA RICA Y SU CONSUMO DE MEDIOS DE COMUNICACIÓN.

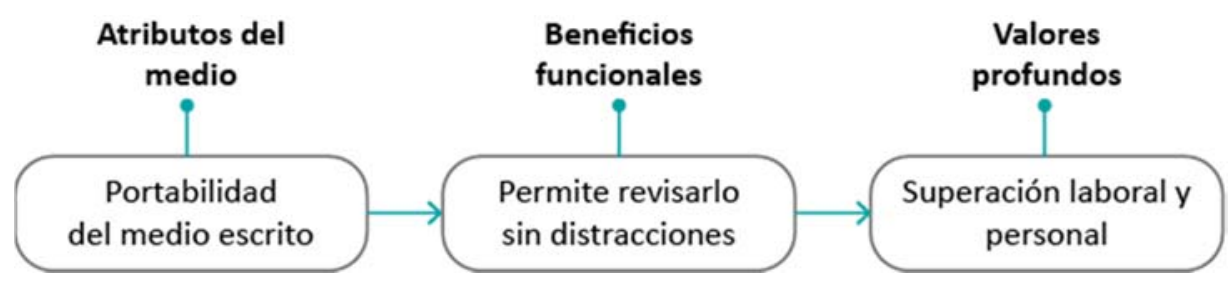

Figura 8. Ejemplo de cadena de medios-fines para medios de comunicación dirigidos a TCO.

Fuente: Elaboración propia.

A pesar de ser un grupo con características demográficas disímiles, su fuerte estructura de gremio les da afinidad en su situación laboral, y esto a su vez, crea coincidencias en los beneficios que buscan y en sus valores (fines). De ahí que, todos los atributos descritos por la muestra como deseables en un medio de comunicación deberán considerar los desafíos de un trabajo físicamente demandante, un horario laboral que inicia a tempranas horas del día, la necesidad de desplazarse largas distancias o trasladar su residencia, un salario relativamente bajo, entre otros.

De ahí que, los temas relacionados con la construcción sean bienvenidos en formatos escritos y que los mensajes en televisión deban ser cortos y llamativos, y que no irrumpan la dinámica doméstica. De manera semejante, al tratarse de un gremio que reconoce las jerarquías, resulta recomendable utilizar el modelado para promover el aprendizaje indirecto. Esto supone el involucramiento de líderes de opinión y la transmisión de información de "boca a boca".

En síntesis, cualquier iniciativa de mercadeo dirigida a esta población que tenga en cuenta estas conexiones tendrá mayores posibilidades de ser aceptada por este segmento. Es recomendable dar particular atención a las consecuencias funcionales y percibidas, así como los valores profundos descritos a lo largo de la investigación, ya que estos son transferibles para cualquier tipo de estrategia y canal de comunicación.

La distribución es quizás el mayor reto encontrado. Sissors y Baron (2002) subrayan que el periódico solamente permite la segmentación geográfica por un muy alto costo. Esto puede poner presión sobre el retorno de la inversión de una publicación impresa dedicada a un segmento relativamente pequeño, pero con alta movilización y segregación geográfica. Adicional a esto, la etapa de introducción está caracterizada por una alta inversión y pocas ganancias (Stanton, Etzel y Walker, 2000). 
TRABAJADORES DE LA CONSTRUCCIÓN EN COSTA RICA Y SU CONSUMO DE MEDIOS DE COMUNICACIÓN.

Es posible que el creciente aumento en la utilización de medios digitales ofrezca opciones de distribución más eficientes, sin embargo, hace falta mayor investigación en el segmento de los TCO, quienes tiene características muy diferentes a las de otros perfiles ocupacionales en la industria de la construcción.

En lo que respecta a la sustentabilidad económica de un medio de comunicación dirigido a los TCO, conviene considerar la experiencia del periódico Manos a la Obra y la revista Tecnocon. Al momento de la investigación, ambos medios recibían ingresos por pauta publicitaria. Sin embargo, ambos experimentaban dificultades debido a que las empresas patrocinadoras cuestionaban el rol de los TCO como decisores en los presupuestos de los proyectos de construcción, y no tenían claro el beneficio de su patrocinio (Godínez, comunicación personal, marzo 2016; J. Alvarado, Comunicación personal, mayo 2010). Teniendo esto en cuenta, es recomendable incluir en la estrategia de marketing, un programa de comunicación activa enfocado en evidenciar el retorno de efectividad y la importancia del segmento, para mantener el interés tanto de los lectores del periódico, como de las empresas anunciantes y la Junta Directiva de las instituciones responsables de su producción. Esto es fundamental para alargar el ciclo de vida de las publicaciones.

\section{REFERENCIAS BIBLIOGRÁFICAS}

Alonso Rivas, J. (2001). Comportamiento del consumidor. Madrid, España: ESIC Editorial.

Azhar, S., y Abeln, J. M. (2014). Investigating social media applications for the construction industry. Procedia Eng. 85: 42-51. doi: 10.1016/j.proeng.2014.10.527.

Azhar, S., Riaz, Z. y Robinson, D. (2019). Integration of Social Media (SM) in day-to-day operations of construction firms. Journal of Management in Engineering. 35. doi: 10.1061/(ASCE)ME.1943-5479.0000660.

Baldi, C. M. y Obando, E. (1998). La condición sociolaboral del migrante nicaragüense en el sector construcción (Tesis de pregrado inédita). Universidad de Costa Rica, San José, Costa Rica.

Baruah, B. (2010). Women and globalization: challenges and opportunities facing construction workers in contemporary India. Development in Practice, (20), 31-44.

Cunradi, C., Ames, G. y Moore, R. (2008). Prevalence and Correlates of Intimate Partner Violence Among a Sample of Construction Industry. Springer Science + Business Media, (23) 101110. 
TRABAJADORES DE LA CONSTRUCCIÓN EN COSTA RICA Y SU CONSUMO DE MEDIOS DE COMUNICACIÓN.

Hawkins, D. I., Best, Roger J. y Coney, K. A. (2004). Comportamiento del consumidor: construyendo estrategias de marketing. México: McGraw-Hill Interamericana.

Hernández Sampieri, R., Fernández, C., y Baptista, P. (2006). Metodología de investigación. México: McGraw-Hill Interamericana.

lacuone, D. (2005). "Real Men Are Tough Guys": Hegemonic Masculinity and Safety in the Construction Industry. The Journal of Men's Studies, (13) 247-265.

Instituto Nacional de Estadística y Censos. (2011) Población ocupada de 15 años y más por grupos de edad, según provincia, sexo y rama de actividad (grupo mayor). Recuperado de https://www.inec.cr/empleo/temas-especiales-de-empleo

Kotler, P. y Armstrong, G. (2013) Fundamentos de marketing. México: Pearson Educación.

Loudon, D. y Della Bitta, A. (1995) Comportamiento del consumidor: conceptos y aplicaciones. México: McGraw-Hill.

Ministerio de Trabajo y Seguridad Social de Costa Rica (2009) Consejo Nacional de Salarios Resolución Administrativa No 03-2000. Perfiles Ocupacionales. Recuperado de http://www.mtss.go.cr/elministerio/consejostripartitosydialogosocial/consejo-nacional-desalarios/documentos\%20consejo\%20nacional\%20de\%20salarios/PERFILES_OCUPACIO NALES_\%20Resolucion_03-2000.pdf.

Perera, S., Victoria, M. y Brand, S. (2017). Social Media in Construction: An Exploratory Case Study. Advances in construction ICT and e-business. doi: 10.4324/9781315690698-16.

Peter, J. P. y Olson, J.C. (2006). Comportamiento del consumidor y estrategia de marketing $\left(7^{\mathrm{a}}\right.$. Ed.). México: McGraw-Hill.

Pride, W., y Ferrell, O. (1997). Marketing: conceptos y estrategias. México: McGraw-Hill.

Real Academia Española. (s.f.), Diccionario de la lengua española, (23a ed.). Recuperado de https://dle.rae.es/gusto?m=form el 13 de marzo de 2021.

Real Academia Española. (s.f.), Diccionario de la lengua española, (23a ed.). Recuperado de https://dle.rae.es/hábito?m=form el 13 de marzo de 2021.

Real Academia Española. (s.f.), Diccionario de la lengua española, (23a ed.). Recuperado de http://lema.rae.es/drae/?val=preferencia el 13 de Marzo de 2021.

Schiffman, L.G. y Kanuk, L. L. (2005). Comportamiento del consumidor. México: Pearson Education, Inc.

Sissors, J. y Baron, R. (2002). Advertising Media Planning. Chicago: McGraw-Hill.

Stanton, W. J., Etzel, M. J. y Walker, B. J. (2000). Fundamentos de Marketing. México: McGrawHill. 
TRABAJADORES DE LA CONSTRUCCIÓN EN COSTA RICA Y SU CONSUMO DE MEDIOS DE COMUNICACIÓN.

Tang, L., Griffith, L., Stevens, M. y Hardie, M. (2020), Social media analytics in the construction industry comparison study between China and the United States. Engineering, Construction and Architectural Management. Vol. 27 No. 8, 1877-1889. doi: 10.1108/ECAM-12-20190717

Unidad de Estadística de La Organización de las Naciones Unidas. (2009) Clasificación Industrial Internacional Uniforme de todas las actividades económicas (CIIU). Recuperado de http://unstats.un.org/unsd/publication/SeriesM/seriesm_4rev4s.pdf

Zhou, D. y Zhou, J. (2009). An Investigation into the Group Characteristics of Casual Construction Workers. Chinese Sociology and Anthropology. (41) 31-39. 